\section{Nanomedicine to Tumor by Enzymosomes}

\section{Yallappamaharaj R Hundekar', Basavaraj K Nanjwade ${ }^{2 *}$, Anas S Mohamied ${ }^{3}$, Nagi F Idris ${ }^{3}$, Teerapol Srichana ${ }^{4}$ and Azam Sharif Md Shafioul ${ }^{5}$}

${ }^{1}$ Department of Quality Assurance, Elegant Drugs Pvt Ltd., Chalmatti, Karnataka, India

${ }^{2}$ Faculty of Pharmacy, Department of Pharmaceutics, Omer Al-Mukhtar University, Tobruk, Libya

${ }^{3}$ Faculty of Pharmacy, Department of Pharmaceutics, Omer Al-Mukhtar University, Al-Bayda, Libya

${ }^{4}$ Faculty of Pharmaceutical Sciences, Drug Delivery System Excellence Centre, Prince of Songkla University, Hat-Yai, Songkla, Thailand

${ }^{5}$ Chemical Kinomics Research Centre, Future Convergence Research Division, Korea Institute of Science and Technology (KIST), Seoul, South Korea

\begin{abstract}
Usually the enzymes have played a role in enhancing prodrug activation for active targeting by an antibody. In routine practice the use of enzymes is a difficult task due to the loss of activity by their degradation, although they do not have a capacity of penetrating into biological membrane, such a task is handled by placing the enzymes in suitable location i.e., encapsulating an enzyme to the surface of the lipid vesicles or surface of liposomes are called enzymosomes, thus the covalent link of enzymes will minimize alterations of the activity of enzymes, enhances half-life and achieved enzyme activity at targeted site such as tumour cell. The review article includes preparation techniques, characterization and stability of liposomal enzymes drug delivery system.
\end{abstract}

Keywords: Encapsulation; Enzymosomes; Liposomal enzymes; Nanomedicine; Prodrug; Tumour

\section{Introduction}

Nanomedicine is the term used to describe the use of molecular particles to administer by heat, drugs, light or other agents to treat ailing cells within the body. Nanoparticles would have to be manufactured on a nanoscale level which would require tools small enough to work within the nano-scale of measurement [1].

*Corresponding author: Basavaraj $\mathrm{K}$ Nanjwade, Faculty of Pharmacy, Department of Pharmaceutics, Omer Al-Mukthar University, Tobruk, PO Box 919 Libya, Tel: +218 944828793; E-mail: nanjwadebk@gmail.com

Citation: Hundekar YR, Nanjwade BK, Mohamied AS, Idris NF, Srichana T, et al. (2015) Nanomedicine to Tumor by Enzymosomes. J Nanotechnol Nanomed Nanobiotechnol 2: 004.

Received: January 05, 2015; Accepted: February 27, 2015; Published: March 13,2015
Enzymosomes are the part of vesicular drug delivery system, enzymo means enzymes and somes means cell-like, these are liposomal constructs engineered to provide a mini bioenvironmental in which enzymes are covalently immobilized or coupled to the surface of liposomes [2]. Most of the catalytic enzymes are useful in enhancing the potent activity of antitumor drugs, such enzymes are alkaline phosphatase, carboxypeptidase, $\beta$-glucosidase, $\beta$-lactamase. The enzyme reaction specificity provides the prodrug activation at the tumour site, through prior enzyme targeting by using liposomes or via enzyme expressing gene delivery in to the tumour cells [3].

Present study, delivery methods for an enzyme/prodrug strategy can be divided into two major classes: (a) delivery of genes that encode prodrug-activating enzymes into tumour tissues (Gene-Directed Enzyme Prodrug Therapy (GDEPT), Virus Directed Enzyme Prodrug Therapy (VDEPT), etc.); and (b) delivery of active enzymes onto tumour tissues (Antibody-Directed Enzyme Prodrug Therapy (ADEPT) [4].

For the targeting of cytotoxic and antiviral agents to specific cell types is the prodrug activation by antibody enzyme conjugates (known as prodrug activation therapy, ADEPT or Antibody-Directed Catalysis (ADC) [5]. The cancer chemotherapy is limited by lack of specificity toward the tumour cells. This problem has been solved by ADEPT also it is known as an alternative for conventional cancer therapy [6]. In this approach an antibody linked by an enzyme that binds to an antigen preferentially expressed on tumour cells (Figure 1). Subsequent administration of a prodrug results in appropriate conversion of the prodrug into the parent cytotoxic drug at the tumour site. The converted prodrug should kill not only the tumour cells to which the conjugate shave bound but also neighbouring tumour cells [7]. The prodrug is designed to be a substrate for the chosen enzyme (Figure 2).

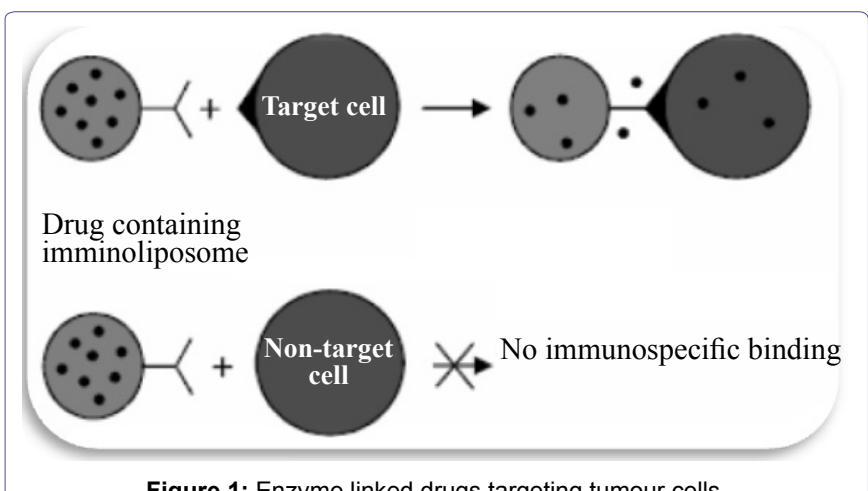

Figure 1: Enzyme linked drugs targeting tumour cells.

The enzyme-activating prodrug therapy in a two-step approach. First step, a drug-activating enzyme is targeted and expressed in tumours. In the second step nontoxic prodrug called as a substrate of the exogenous enzyme is now expressed in tumours and it is administered by parenteral route [2].

The prodrug activation generates a very small toxic species which is not affected by the neutralising action of serum enzymes and may 


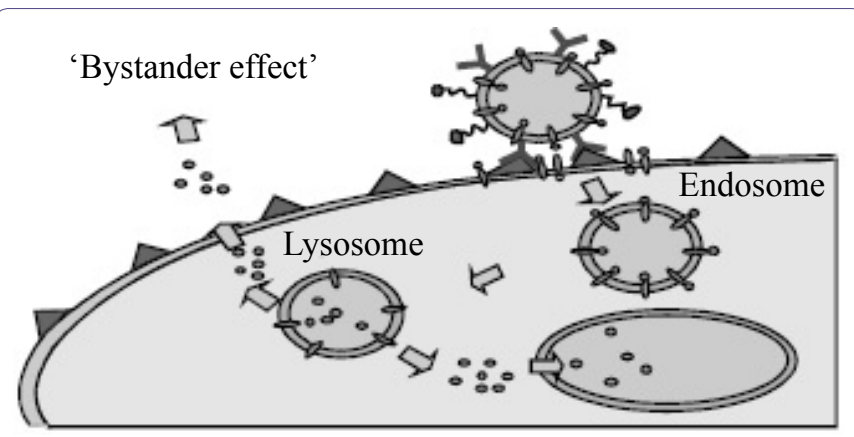

Figure 2: Bystander killing effect.

have a half-life long enough to have a by stander killing effect. This is the cyanide generation system devised by Rowlinson-Busza in 1992 (antibody guided enzyme nitrile therapy) [8].

In tumour therapeutics dun the various body immune components are recognised for attack of disease i.e., tumour antigens (vaccine), antibodies, Monoclonal Antibodies (MAb), cytokinase and immunotoxins etc.

To avoid heterogeneity problem in antigen expression the MAb-guided therapy is useful by using antibodies to carry enzymes to tumour cells. There is huge number of different types of enzymes used in the conversion of prodrugs into respective active drug by coupling with antigen/antibody. Such enzymes are Carboxypeptidase G2, $\beta$-lactamase, $\beta$-glucuronidase, $\beta$-galactosidase, Alkaline Phosphatase, Penicillin V-amidase, Cytosine Deaminase, Nitroreductase, Carboxypeptidase A and catalytic antibodies. Followed by the activity of enzymes is the release of active drug required at the targeted tumour site. Lastly, heterogeneity in antigen expression will result in escape of antigen-negative cells from treatment [2].

The main objective is to develop and optimize enzymosomes, with long circulation times in the blood in order to accumulate at target tumour site, while maintaining enzymatic activity in its intact form. Therefore, the present review work is designed to study of enzymosomes for which enzyme release from the lipidic particles is not needed and simultaneously being able to exert therapeutic effect even when the liposomes remain intact within the pathological site of action. Currently enzymes used for prodrug activation systems with antigen/antibody showing in table1 [9].

contrast with a huge number of publications with antibodies attached to carriers concept widely used for the active targeting of nanocarriers. Superoxide Dismutase (SOD) is used as a therapeutic agent for oxidative stress related diseases such as Rheumatoid Arthritis and Ischaemia/Reperfusion situations. Our objective was to develop and optimize SOD enzymosomes, with long circulation times in the blood in order to accumulate at inflamed target sites, while maintaining enzymatic activity in its intact form [10].

Enzymes are promising therapeutic agents due to their specific substrate selectivity and unparalleled reaction efficiency. The intrinsic shortcomings of enzymes, relatively low enzymatic activity under physiological conditions and short circulation life (Figure 3). An inherent immunogenicity and might be overcome by encapsulating them into certain biocompatible and biodegradable drug carriers, such as lipid vesicles [11].

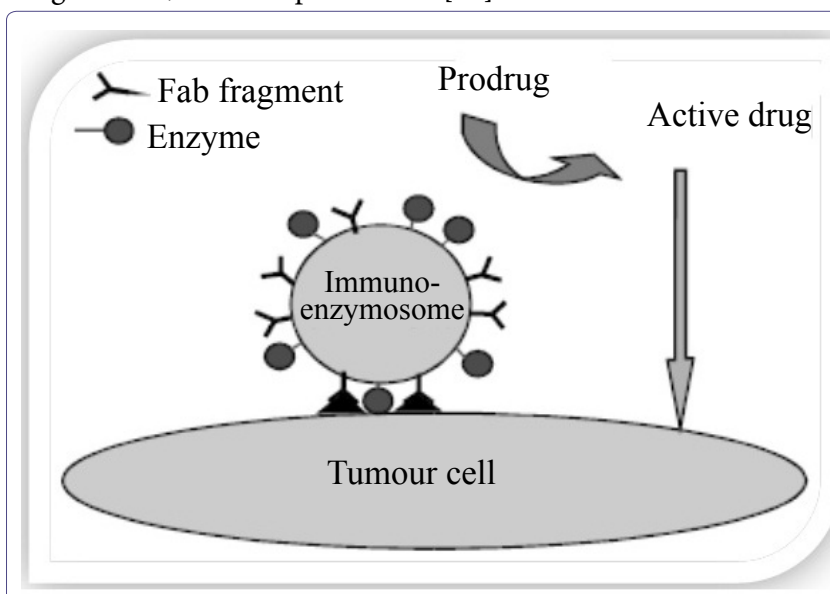

Figure 3: Prodrug converted in to active drug by enzymes.

\section{Advantages of Antibody Directed Enzyme Prodrug}

Therapy

1. A single enzyme can convert a large number of prodrugs to the cytotoxic agents; therefore, the drug potency should not be a problem.

2.The antibody-enzyme complex and prodrug are relatively non-toxic unlike the radionuclide and they should not cause damage when they are in the systemic circulation.

3.Like radionuclide can also overcome the problems of antigenic heterogeneity and tumor accessibility [9].

\begin{tabular}{|c|c|c|c|}
\hline Antibody/Antigen & Enzyme & Prodrug & Active drug \\
\hline MAbs W14A and SBIO/chorionic onadotropin & Carboxypeptidase G2 & Benzoic acid mustards-glutamic acid & Benzoic acid mustards \\
\hline No antibody in study & $\beta$-Lactamase & Vinca-cephalosporin & Vinca alkaloid \\
\hline MAb L6 (against a carbohydrate antigen on human carcinomas) & Penicillin amidase & $\begin{array}{l}\text { Doxorubicin-phenoxyacetamide } \\
\text { Melphalan-phenoxyacetamide }\end{array}$ & Doxorubicin Melphalan \\
\hline MAb BW431/26/Carcinoembryonic Antigen (CEA) & Alkaline phosphatase & Etoposide phosphate & Etoposide \\
\hline MAb 323/A3 (against a pan-carcinoma membrane glycoprotein) & $\beta$-Glucuronidase & Epirubicin-glucoronide & Epirubicin \\
\hline MAb KS1/4/UCLA-P3 human lung adenocarcinoma & Carboxypeptidase A & Methotrexate-alanine & Methotrexate \\
\hline
\end{tabular}

Table 1: Currently used prodrug activation systems with antigen/antibody.

Different strategies can be used to improve carrier mediated delivery of therapeutic proteins as: its incorporation into polymeric carriers, into aqueous space of lipid, detergent or lipid-detergent vesicles or incorporation of hydrophobized ones into lipid bilayer of vesicles. A strategy not usually used for therapeutic enzymes. It is attachment to the outer surface of liposomes and using technologies developed for antibodies. Some publications report the construction of carriers with surface attached enzymes in

\section{Disadvantages of Antibody Directed Enzyme}

\section{Prodrug Therapy}

ADEPT will result in toxicity in normal cells, if:

a. The body itself has enzymes that can convert the prodrug to the cytotoxic agent.

b. A residual amount of enzyme-antibody conjugates remains in the systemic circulation 
c. The produced cytotoxic agent at the tumor site gets back to the systemic circulation.

d.The tumor specific antibody has cross reactivity with the cell surface antigens of normal cells and delivers the enzyme to the normal tissue [12].

\section{Liposome Preparation}

Mainly three different liposome preparation methods for the encapsulation of enzyme SOD in long circulating liposomes [13].

\section{Film hydration method, pH 5.6}

MLV were prepared by the appropriate amounts of E-PC: Chol: DSPE-PEG in a required molar ratio in chloroform was dried under a nitrogen stream until a homogeneous film was formed. Prepared film was dispersed in a solution of SOD in $\mathrm{NaCl}$ citrate buffer $\mathrm{pH} 5.6$ (required lipid concentration) [14].

\section{Freeze-thawing method, pH 5.6}

Freeze-thawing was prepared by E PC: Chol: DSPE-PEG in a required molar ratio in chloroform was dried under a nitrogen stream until a homogeneous film was formed. Prepared film was dispersed in a solution of SOD in $\mathrm{NaCl}$ citrate buffer $\mathrm{pH} 5.6$ (it required lipid concentration). Five cycles of freezing in liquid nitrogen $(5 \mathrm{~min})$ followed by thawing at $37 \mathrm{jC}(5 \mathrm{~min})$ concluded this protocol [15].

\section{Dehydration and rehydration method, pH 5.6}

In this method which the appropriate amounts of EPC: Chol: DSPE-PEG in a required ratio in chloroform was dried under a nitrogen stream until a homogeneous film was formed. Prepared film was dispersed in a solution of SOD in water (it required lipid concentration), frozen in liquid nitrogen and lyophilized overnight. Then mannitol citrate buffer $\mathrm{pH} 5.6$ was added to the lyophilized powder up to $1 / 10$ of the volume of the original dispersion. Then hydration step lasted $30 \mathrm{~min}$, and, subsequently, $\mathrm{NaCl}$ citrate buffer pH 5.6 were added to reach the starting volume. Then except for the dispersions that were extruded, nonencapsulated protein was separated from the liposome dispersion by dilution and ultracentrifugation. Finally, liposomes were dispersed in a $\mathrm{NaCl}$ citrate buffer $\mathrm{pH} 5.6$ [16]

\section{Conjugation of Enzyme SOD to Liposomes for In Vivo Studies}

The conjugation of enzyme SOD to liposomes containing the lipid anchors MPB-PE or Maleimide-PEG-PE was performed. The thioacetylation reagent, SATA, in dimethylformamide was mixed with the enzyme in a buffer solution. The thioacetylated enzyme, SOD-ATA, after separation from unreacted SATA on a PD-10 column, was de-acetylated with hydroxylamine. The thiolated enzyme, SOD-AT was added to the liposomes containing the reactive groups MPB or maleimide. Then enzymosomes were separated by ultracentrifugation and suspended in buffer [17].

\section{Liposome Characterization}

The liposomal formulations has been characterized by lipid composition, shape, size and other parameters like -incorporation efficiency, initial and final protein to lipid ratio (Prot/Lip), zeta potential and enzymatic activity (retention of enzymatic activity and externally exposed enzyme activity) [10].

\section{Physicochemical Characterization}

An adequate physicochemical characterization of the final SLN dispersions is of crucial importance but due to the small size, complexity and-normally-heterogeneity of dispersions not an easy task [18]. The main points of interests physicochemical state, size and size distribution, surface properties of the nanoparticles, shape of the nanoparticles, presence of additional colloidal structure (e.g., due to the excess of stabilizer) and drug localization (nanoparticle core, interface additional structures, precipitated drug).

\section{Size and zeta potential}

Size and surface properties are the most important properties of nanoparticles since they are the main determinants for the performance of the drug carrier system in vivo. The most commonly used method for size determination of colloidal particles is Photon Correlation Spectroscopy (PCS) but methods based on static light scattering (laser diffraction with suitable instrumentation for sub-micron range) can give additional information about particles in the $\mu \mathrm{m}$-range and size distribution. A further, not yet often used method presents the Asymmetrical Flow Field-Flow Fraction (A4F) combined with multi-angle light scattering. By this method accurate information about the size distribution can be obtained due the separation of the nanoparticles before size determination.

\section{Photon Correlation Spectroscopy (PCS)}

Photon correlation spectroscopy-also Dynamic Light Scattering (DLS) or Quasi-Elastic Light Scattering (QELS) is a robust and rapid method for size determinations in the colloidal range (from about $5 \mathrm{~nm}$ to $1 \mu \mathrm{m})$. In PCS, intensity fluctuations of the scattered light due to particle motion are measured in dependence on time. By the diffusion coefficient of the particles in the measurement fluid, the particle diameter can be calculated according to the Stoles-Einstein equation.

\section{Laser diffraction with sub-micron equipment}

Additionally to PCS, laser diffraction where the intensity of scattered light is measured in dependence on the angle (Static light scattering) is most commonly used for size determinations of SLN. Classical laser diffraction (Fraunhofer diffraction) can only be meaningfully applied for particles with a size larger than the wavelengths of the laser light used for the measurements (normally $633 \mathrm{~nm})$.

\section{Asymmetrical Flow Field-Flow Fraction (A4F)}

Asymmetric flow field-flow fractionation was used for size determinations of SLN in comparison to an emulsion and oil-loaded SLN. The differences found in the sizes and elution profiles were attributed to differences in the particle shapes. Due to their anisometric, platelet-like shape it is likely that SLN are more retained by the cross flow applied compared to spherical emulsion droplets. This method appears very promising as additional size determination method particularly with regard to separation and detection of different colloidal structures.

\section{Zeta potential}

From zeta potential measurements information about the surface charge of the nanoparticles can be derived. A sufficient high zeta potential improves the stability of electrostatically stabilized nanoparticles due to the increased particle repulsion by electrostatic 
Citation: Hundekar YR, Nanjwade BK, Mohamied AS, Idris NF, Srichana T, et al. (2015) Nanomedicine to Tumor by Enzymosomes. J Nanotechnol Nanomed Nanobiotechnol 2: 004 .

- Page 4 of 5 •

\begin{tabular}{|c|c|c|}
\hline Vesicular System & Description & Application \\
\hline Enzymosomes & $\begin{array}{l}\text { Liposomal construct engineered to provide a mini bio environment in which the enzyme covalently immobilized to } \\
\text { the surface of liposomes. }\end{array}$ & Targeted delivery to tumor cell. \\
\hline Virosomes & $\begin{array}{l}\text { Liposomes spiked with virus glycoprotein's, incorporated in the liposomal bilayer based on retrovirus based } \\
\text { lipids. }\end{array}$ & Immunological adjuvants. \\
\hline Ufasomes & $\begin{array}{l}\text { Vesicles enclosed by fatty acids obtained by long chain fatty acids by mechanical agitation of evaporated film in } \\
\text { the presence of buffer solution. }\end{array}$ & Ligand mediated drug targeting. \\
\hline Cryptosomes & $\begin{array}{l}\text { Lipid vesicle with surface coat composed of PC and of suitable polyoxyethylene derivative of phosphatidyl } \\
\text { ethanolamine. }\end{array}$ & Ligand mediated drug delivery. \\
\hline Emulsomes & Nanosized lipid particles consisted of lipid assembly and a polar group. & $\begin{array}{l}\text { Parenteral delivery of poorly water } \\
\text { soluble drugs. }\end{array}$ \\
\hline Discosomes & Niosomes coupled with non-ionic surfactants. & Ligand mediated drug targeting. \\
\hline Aquasomes & $\begin{array}{l}\text { These are spherical } 60-300 \mathrm{~nm} \text { particles used for drug and antigen delivery. The particle core is composed of } \\
\text { non crystalline calcium phosphate or ceramic diamond and is covered by a polyhydroxyloligomeric film. }\end{array}$ & $\begin{array}{l}\text { Specific targeting, molecular } \\
\text { shielding. }\end{array}$ \\
\hline Collidosomes & $\begin{array}{l}\text { Are solid microcapsules formed by the self assembly of colloidal particles at the interface of emulsion droplets? } \\
\text { "Colloidosomes" are hollow, elastic shells whose permeability and elasticity can be precisely controlled. }\end{array}$ & Drug targeting. \\
\hline Genosomes & Artificial macromolecular complex for functional gene transfer. & Cell specific gene transfer. \\
\hline Photosomes & $\begin{array}{l}\text { Photolyase encapsulated in liposomes, which release the contents by photo triggered charges in membrane } \\
\text { permeability characteristics. }\end{array}$ & Photodynamic therapy. \\
\hline Erythrosomes & $\begin{array}{l}\text { Liposomal system in which chemically cross-linked human erythrocytes cytoskeletons are used as to which a } \\
\text { lipid bilayer is coated. }\end{array}$ & Targeting of macromolecular drugs. \\
\hline Hemosomes & Heamoglobin containing liposomes engineered by immobilizing heamoglobin with polymerizable phospholipids. & $\begin{array}{l}\text { High capacity oxygen carrying } \\
\text { system. }\end{array}$ \\
\hline Protosomes & High molecular weight multi subunit enzyme complexes with catalytic activity. & $\begin{array}{l}\text { Better catalytic activity turnover than } \\
\text { non associated enzymes. }\end{array}$ \\
\hline Vesosomes & Nested bilayer composed of bilayer. & $\begin{array}{l}\text { Multiple compartments of vesosomes } \\
\text { give better protection to the interior } \\
\text { content of serum. }\end{array}$ \\
\hline Archaeosomes & Vesicles composed of gylcerolipids of archaea with potent adjuvant activity. & Poor adjuvant activity. \\
\hline Cubosomes & $\begin{array}{l}\text { Are bicontinuous cubic phases, consisting of two separate, continuous, but non intersecting hydrophilic regions } \\
\text { divided by a lipid layer that is contorted into a periodic minimal surface with zero average curvature. }\end{array}$ & Drug targeting. \\
\hline
\end{tabular}

Table 2: Comparative overview of enzymosome with current emerging vesicular system.

forces. However, this rule cannot be strictly applied for formulations stabilized with polymers resulting in a steric stabilization.

\section{Pharmacokinetics and Pharmacodynamics (PK/PD)}

Data collected at multiple time points after the administration of a nanomedicine in animal models allows for a complete pharmacokinetics and pharmacodynamic analysis of the nanoagent in vivo. $\mathrm{PK} / \mathrm{PD}$ assessments are performed using WinNonlin data management a statistics, modelling and visualization tool for PK data analysis. Parameters typically provided for given nanomedicine include drug concentration over time, Area Under the Curve (AUC), elimination half life, clearance, and time of maximum concentration [19].

\section{Stability Studies}

The free catalase thermal stability is dependent on its concentration. The higher enzyme concentration may higher its thermal stability i.e., $0.25 \mathrm{mg} / \mathrm{ml}$ to $5.0 \mathrm{mg} / \mathrm{ml}$. This means dissociation of the enzyme into its subunits dominates the enzyme deactivation. On the other hand, the catalase at the highest concentration i.e., $16 \mathrm{mg} / \mathrm{ml}$ showslower stability than that at $5.0 \mathrm{mg} / \mathrm{ml}$. For the $16 \mathrm{mg} / \mathrm{ml}$ of catalase, the formation of irreversible intermolecular aggregates is facilitated among the conformationally altered enzyme molecules. Quite importantly, the thermal stability of the liposomal catalase with in of $16 \mathrm{mg} / \mathrm{ml}$ (CAL100-III) is much higher than that of free enzyme at the identical concentration. This is because the enzyme formation aggregatesis prevented in the liposomal aqueous phase through the interaction of the inner surface of the liposome membrane with the encapsulated enzyme molecules. Therefore the functional carrier liposomal system has the stabilization effect on the structure and activity of the liposome-encapsulated catalase molecules [20].

\section{Regulatory Aspects}

The design and development of engineered nanosystems for disease diagnosis, prevention and treatment has been boosted by significant advances observed in distinct areas. This has been combined in order to attain efficient and safe products. Even if some of those nanoproducts have successfully reached the market, the consensus underlying the nanomedicines-related regulatory requirements has still to be fully acquired by part of the scientific community and thus academia and pharmaceutical companies could be facing considerable obstacles and during the research and development life cycle of these medicinal products. However, considerable progress has been made in the last years, reflecting the recognition by regulatory authorities specific features associated to the nanosystems-based medicines. As a consequence the regulatory environment for those innovative medicinal nanosystems has been increasingly challenged by key issues, which has been an opportunity to provide clear guidance for their development [21].

\section{Future Prospective}

Liposomal constructs engineered to provide a mini bioenvironmental in which enzymes are covalently immobilized or coupled to the surface of liposomes. Targeted drug delivery system to tumour cell. For targeting cells of tumour like diseases the enzyme 
Citation: Hundekar YR, Nanjwade BK, Mohamied AS, Idris NF, Srichana T, et al. (2015) Nanomedicine to Tumor by Enzymosomes. J Nanotechnol Nanomed Nanobiotechnol 2: 004 .

containing prodrugs conjugates are helpful and valuable. In various cases the administration of drugs or prodrugs to body via blood circulation may cause somewhat failure by some other pharmacological activity. Also the direct administration of these medications to target cells is somewhat impossible. That's what by using enzyme conjugates with prodrugs may minimise the failureness of target cell treatment also the utilization of complete medicine by appropriate enzyme activity so in next few years such type of enzyme containing life threatening drugs may developed for the sake of treatment of cancerous like diseases [18-21]. Comparative overview of enzymosome with current emerging vesicular system, e.g., Enzymosomes, Virosomes, Ufasomes, Cryptosomes, Emulsomes, Discosomes, Aquasomes, Collidosomes, Genosomes, Photosomes, Erythrosomes, Hemosomes, Protosomes, Vesosomes, Archaeosomes and Cubosomes (Table 2) [22]

\section{Conclusion}

Enzymosomes of vesicular systems depicting their importance; the system provides flexibility for drug design thus overcoming various bioavailability and solubility problems. The significance of the system lies in controlling and sustaining drug action. Though there are number of shortcomings associated with vesicular delivery, still they play an important role in bringing new life to the old drugs (pre-existing). The upcoming new systems are predicted to bring forward the new era of drug delivery.

\section{References}

1. Bhowmik D, Chiranjib, Chandira RM, Tripathi KK, Kumar KPS (2010) Nanomedicine-An overview. International Journal of PharmTech Research 2: 2134-2151.

2. Kumar R, Kumar S, Jha SS, Jha AK (2011) Vesicular System-Carrier for Drug Delivery. Pelagia Research Library 2:192-202.

3. Vyas SP, Khar RK (2012) Immunotherapy of tumour. Targeted \& Controlled Drug Delivery: Novel Carrier Systems. CBS, India. Pg: 521-523.

4. Deonarain MP, Epenetos AA (1994) Targeting enzymes for cancer therapy: old enzymes in new roles. Br J Cancer 70: 786-794.

5. Fonseca MJ, Jagtenberg JC, Haisma HJ, Storm G (2003) Liposome-mediated targeting of enzymes to cancer cells for site-specific activation of prodrugs: comparison with the corresponding antibody-enzyme conjugate. Pharm Res 20: $423-428$.

6. Vingerhoeds $M H$, Haisma HJ, van Muijen M, van de Rijt RB, Crommelin DJ, et al. (1993) A new application for liposomes in cancer therapy. Immunoliposomes bearing enzymes (immuno-enzymosomes) for site-specific activation of prodrugs. FEBS Lett 336: 485-490.

7. Xu G, McLeod HL (2001) Strategies for enzyme/prodrug cancer therapy. Clin Cancer Res 7: 3314-3324.
8. Haisma HJ, Boven E, van Muijen M, de Jong J, van der Vijgh WJ, et al. (1992) A monoclonal antibody-beta-glucuronidase conjugate as activator of the prodrug epirubicin-glucuronide for specific treatment of cancer. $\mathrm{Br} \mathrm{J}$ Cancer 66 : 474-478.

9. Foldvari M, Jaafari MR, Mezei M, Mezei C (1998) Targeting of liposomes through immunoglobulin superfamily domains: P0 protein as a model. Drug Deliv 5: 183-195.

10. Vale CA, Corvo ML, Martins LCD, Marques CR, G Storm, et al. (2006) Construction of Enzymosomes: Optimization of Coupling Parameters. NSTI-Nanotech 2: 396-397.

11. Tan QY, Zhang JQ, Wang N, Yang H, Li X, et al. (2012) Improved biological properties and hypouricemic effects of uricase from candida utilis loaded in novel alkaline enzymosomes. Int J Nanomedicine 7: 3929-3938.

12. Corvo ML, Jorge JC, van't Hof R, Cruz ME, Crommelin DJ, et al. (2002) Superoxide dismutase entrapped in long-circulating liposomes: formulation design and therapeutic activity in rat adjuvant arthritis. Biochim Biophys Acta 1564: 227-236.

13. Biel S, Simon J, Gross R, Ruiz T, Ruitenberg M, et al. (2002) Reconstitution of coupled fumarate respiration in liposomes by incorporating the electron transport enzymes isolated from Wolinella succinogenes. Eur J Biochem 269: 1974-1983.

14. Riaz M (1996) Liposomes preparation methods. Pak J Pharm Sci 9: 65-77.

15. Traïkia M, Warschawski DE, Recouvreur M, Cartaud J, Devaux PF (2000) Formation of unilamellar vesicles by repetitive freeze-thaw cycles: characterization by electron microscopy and 31P-nuclear magnetic resonance. Eur Biophys J 29: 184-195.

16. Gaspar MM, Boerman OC, Laverman P, Corvo ML, Storm G, et al. (2007) Enzymosomes with surface-exposed superoxide dismutase: In vivo behaviour and therapeutic activity in a model of adjuvant arthritis. Journal of Control Release 117: 186-195.

17. Ruozi B, Belletti D, Tombesi A, Tosi G, Bondioli L, et al. (2011) AFM, ESEM, TEM, and CLSM in liposomal characterization: a comparative study. Int $J$ Nanomedicine 6: 557-563.

18. Kuntsche J, Mader K (2010) Solid Lipid Nanoparticles (SLN) for Drug Delivery. In: Torchilin V, Amiji MM (eds.). Handbook of Materials for Nanomedicine. Pan Stanford Publishing, United Kingdom. Pg: 403-408.

19. http://ncore.web.unc.edu/in-vivo-pkpd-studies/

20. Yoshimoto M (2011) Stabilization of enzymes through encapsulation in liposomes. Methods Mol Biol 679: 9-18.

21. Gaspar RS, Florindo HF, Silva LC, Videira MA, Corvo ML, et al. (2014) Regulatory Aspects of Oncologicals: Nanosystems Main Challenges. In: Alonso MJ, Garcia-Fuentes M (eds.). Nano-Oncologicals. Springer International Publishing, Switzerland, Pg: 425-452.

22. Rathore P, Swami G (2012) Planterosomes: a potential phyto-phospholipid carriers for the bioavailability enhancement of herbal extracts. IJPSR 3: 737755 . 